\title{
The Crystal Structure of $\alpha-\mathrm{La}_{2} \mathrm{Mo}_{2} \mathrm{O}_{9}$ and the Structural Origin of the Oxide Ion Migration Pathway
}

Ivana Radosavljevic Evans*, Judith A. K. Howard and John S. O. Evans

Department of Chemistry, University of Durham, Science Site, South Road, Durham DHI

3LE, U.K. Fax: +44 191 384737; Tel: +441913342004

*ivana.radosavljevic@durham.ac.uk

\section{Supplementary Data}

\section{Time-of-flight neutron powder diffraction data}

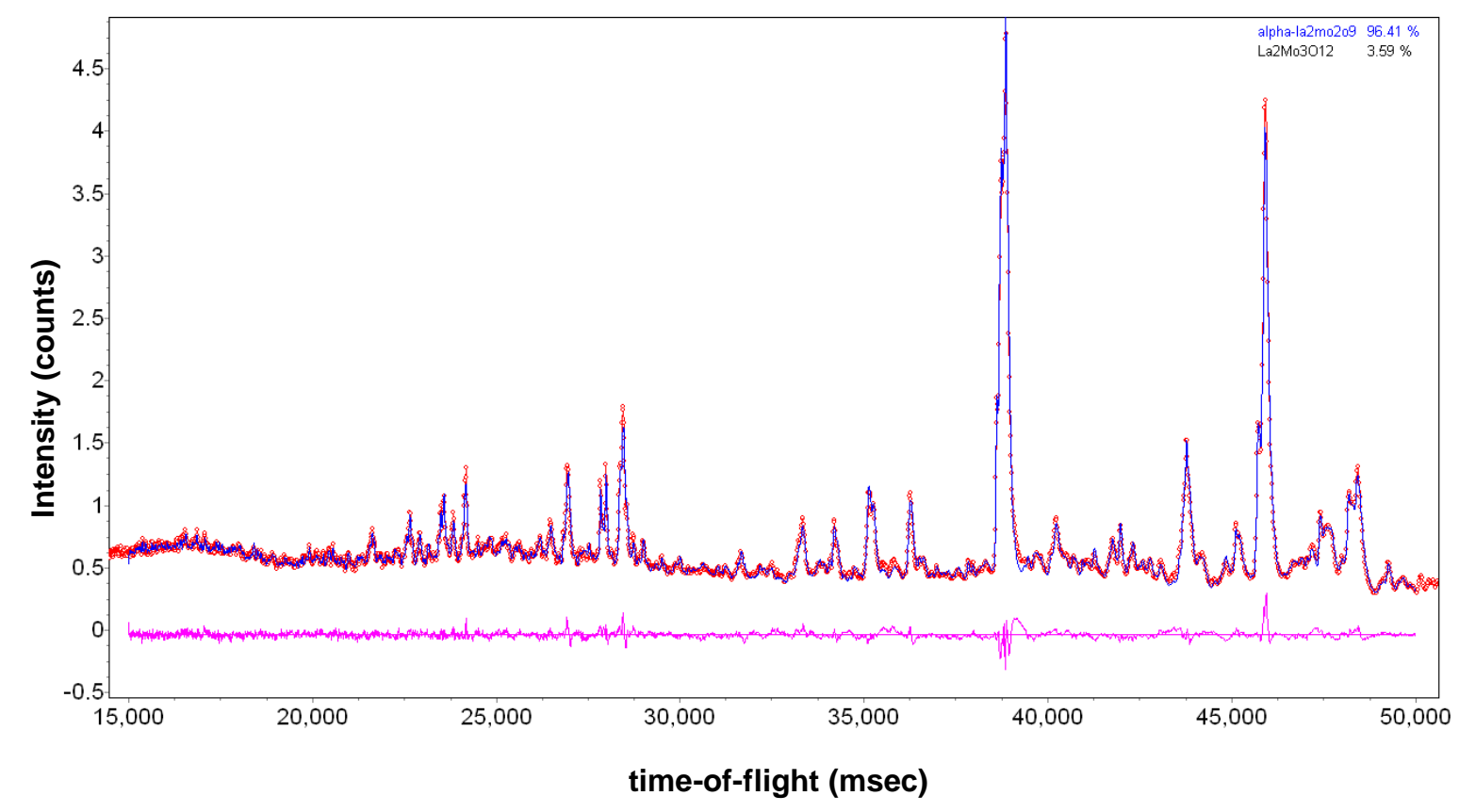

Figure 1: Rietveld fit of the single crystal structural model of $\mathrm{La}_{2} \mathrm{Mo}_{2} \mathrm{O}_{9}$ to neutron powder diffraction data collected on a $10 \mathrm{~g}$ sample using the SIRIUS time of flight diffractometer at 
the High Energy Accelerator Research Organization (KEK). Time of flight range shown corresponds to a d-spacing range of 0.74 to $2.47 \AA$. 36 parameters were refined: 15 background terms, 11 terms describing peak shape, instrumental parameters and scale factors, 4 unit cell parameters and a single overall isotropic temperature factor for $\mathrm{La}_{2} \mathrm{Mo}_{2} \mathrm{O}_{9} ; 4$ unit cell parameters and a single overall isotropic temperature factor for a minor $\mathrm{La}_{2} \mathrm{Mo}_{3} \mathrm{O}_{12}$ impurity, included as a second phase). Final agreement factors obtained: $\mathrm{R}_{\mathrm{wp}}=3.97 \%, \mathrm{R}_{\mathrm{Bragg}}$ $=2.03 \%$.

The authors acknowledge Dr Richard Gover and Dr Takashi Kamiyama of the Institute of Materials Structure Science, High Energy Accelerator Research Organization, Japan, for the assistance with the neutron powder diffraction data collection.

\section{Spread of bond valence sums in the freely refined structure}

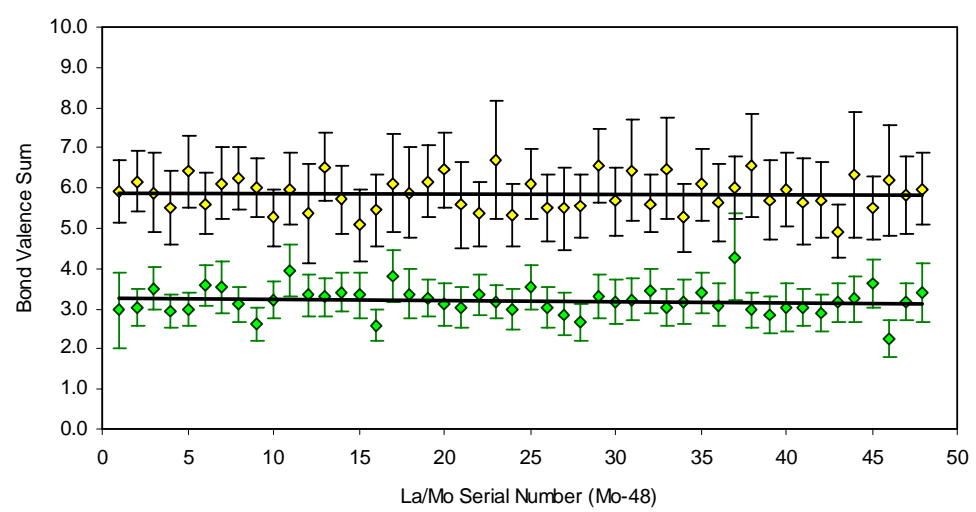

Figure 2: Bond Valence sums for the 96 cations in the freely refined structure (Mo in yellow, $\mathrm{La}$ in green). Error bars are plotted as \pm 1.5 esd's based on standard uncertainties in M-O bond lengths. 